\title{
Innominate artery cannulation: An alternative to femoral or axillary cannulation for arterial inflow in proximal aortic surgery
}

\author{
Ourania Preventza, MD, ${ }^{\mathrm{a}, \mathrm{b}}$ Faisal G. Bakaeen, MD, ${ }^{\mathrm{b}, \mathrm{c}}$ Elizabeth H. Stephens, MD, PhD, \\ Susan M. Trocciola, MD, ${ }^{\mathrm{a}, \mathrm{b}}$ Kim I. de la Cruz, MD, ${ }^{\mathrm{a}, \mathrm{b}}$ and Joseph S. Coselli, MD ${ }^{\mathrm{a}, \mathrm{b}}$
}

Objective: To evaluate the effectiveness of innominate artery cannulation in proximal aortic procedures, including those involving hypothermic circulatory arrest.

\begin{abstract}
Methods: A total of 68 patients underwent innominate artery cannulation with a side graft during proximal aortic surgery performed by way of a median sternotomy. The indications for surgery were proximal arch aneurysm in 43 patients $(63.2 \%)$, aortic dissection in 11 patients $(16.2 \%)$, total arch aneurysm in 10 patients $(14.7 \%)$, and ascending aortic aneurysm in 4 patients $(5.9 \%)$. Six patients $(8.8 \%)$ had undergone previous sternotomy. Hypothermic circulatory arrest with antegrade cerebral perfusion was used in 64 patients $(94.1 \%)$. Of the 68 patients, $63(92.6 \%)$ received antegrade cerebral perfusion to both cerebral hemispheres. The median antegrade cerebral perfusion time was 20 minutes (range, 15.0-33.0 minutes). Seven patients had periods of circulatory arrest without antegrade cerebral perfusion for a median of 20 minutes (range, 6-33 minutes).
\end{abstract}

Results: One patient died, for 30-day mortality of $1.5 \%$. Three patients $(4.4 \%)$ had strokes, two of whom had a partial recovery. Seven patients $(10.3 \%)$ developed temporary postoperative confusion that resolved successfully in all cases.

Conclusions: Cannulating the innominate artery for arterial inflow is an alternative technique for proximal aortic surgery procedures. It is especially useful in cases requiring hypothermic circulatory arrest to deliver antegrade cerebral perfusion. (J Thorac Cardiovasc Surg 2013;145:S191-6)

Because aortic surgery is associated with an increased risk of neurologic complications, there have been many modifications to the surgical techniques and cerebral protection methods to reduce these risks. Hypothermic circulatory arrest (HCA) with antegrade cerebral perfusion (ACP) has been adopted at many centers, including ours. We previously described our technique of axillary artery cannulation to facilitate ACP. ${ }^{1}$ However, axillary artery cannulation necessitates a second incision and entails a risk of brachial plexus and subclavian vein injury. ${ }^{2}$ Innominate artery cannulation was described by Banbury and Cosgrove ${ }^{3}$ in 2000. Since then, modifications have been reported in other case series. ${ }^{2,4}$ The purpose of our present study was to evaluate the safety of innominate artery cannulation in proximal aortic procedures and the effectiveness of delivering ACP during HCA.

\footnotetext{
From the Texas Heart Institute, ${ }^{a}$ St Luke's Episcopal Hospital, Houston, Tex; Division of Cardiothoracic Surgery, ${ }^{\text {b }}$ Michael E. DeBakey Department of Surgery, Baylor College of Medicine, Houston, Tex; and Michael E. DeBakey Veterans Affairs Medical Center, ${ }^{\mathrm{c}}$ Houston, Tex.

Disclosures: Drs Preventza, Bakaeen, Stephens, Trocciola, de la Cruz, and Coselli have nothing to disclose with regard to commercial support.

Presented at the American Association for Thoracic Surgery Aortic Surgery Symposium 2012, April 26-27, 2012, New York, NY.

Received for publication April 20, 2012; revisions received July 31, 2012; accepted for publication Nov 28, 2012; available ahead of print Dec 26, 2012.

Address for reprints: Ourania Preventza, MD, Texas Heart Institute, St Luke's Episcopal Hospital, 6770 Bertner Ave, Suite C-350, Houston, TX 77030 (E-mail: opsmile01@aol.com).

0022-5223/\$36.00

Copyright (c) 2013 by The American Association for Thoracic Surgery

http://dx.doi.org/10.1016/j.jtcvs.2012.11.061
}

\section{METHODS}

The Baylor College of Medicine institutional review board approved our request to collect and analyze the clinical data. The data were collected from a prospectively maintained clinical database by the same team of surgeons involved in the Perioperative care of the patients. All patients provided informed consent for data collection.

\section{Patient Characteristics}

From June 2011 to February 2012, 68 consecutive patients underwent innominate artery cannulation with a side graft during proximal aortic surgery performed by way of a median sternotomy. The group included 50 men and 18 women, with a median age of 61.5 years (interquartile range, 50.5-72.0 years). The indications for surgery are summarized in Table 1 .

\section{Study Design and Variables}

Surgical diagrams and medical records were used to verify the collected data. The overall patient characteristics are listed in Table 2. Follow-up data were obtained from the hospital's medical records department and the Social Security death index.

Cardiac disease unrelated to the aorta was defined as a history of arrhythmia, cardiac surgery, coronary artery disease, or heart failure. Pulmonary disease was defined as a history of restrictive or obstructive lung disease. Genetically triggered thoracic aortic disease included Marfan syndrome, familial thoracic aortic aneurysm, and genetic mutations.

Our primary outcome measure was all postoperative neurologic events, and the secondary outcome measures included operative mortality and postoperative complications. We have previously published our definitions of outcome variables with regard to operative mortality, stroke, and acute renal insufficiency. ${ }^{5}$ In brief, stroke was defined as any new brain injury evident either clinically or radiographically after the procedure. Operative mortality was defined as death within 30 days after operation or before hospital discharge. Acute renal dysfunction included either the need to start dialysis or a doubling of the serum creatinine level. In addition, postoperative confusion and agitation were defined as a temporary neurologic 


\section{Abbreviations and Acronyms \\ $\mathrm{ACP}=$ antegrade cerebral perfusion \\ HCA $=$ hypothermic circulatory arrest \\ NIRS $=$ near-infrared spectroscopy}

deficit with no focal deficit on computed tomography or magnetic resonance imaging scans. The length of hospital stay was determined by the number of days between the procedure and discharge from our facility. The operative times were defined as follows. The myocardial ischemia time was the interval from the initiation of circulatory arrest or crossclamping until clamp removal. The cardiopulmonary bypass time was defined as the period during which the patient was supported by cardiopulmonary bypass, not including the ACP time or cerebral circulatory arrest time. The cerebral circulatory arrest time was the period of circulatory arrest, without ACP. Finally, the ACP time was defined as the period of circulatory arrest during which the patient was receiving ACP.

\section{Surgical Technique of Innominate Artery Cannulation}

After a median sternotomy was performed, the innominate vein was identified and encircled with an umbilical tape, allowing the vein to be retracted inferiorly. The innominate artery was exposed, and dissection was performed to the bifurcation. Exposure was facilitated with an appendiceal retractor. The innominate artery was encircled with an umbilical tape, and $1 \mathrm{mg} / \mathrm{kg}$ of heparin was given. After 3 minutes, a partially occluding crossclamp was applied to the distal innominate artery. An 8-mm Terumo Vascutek Gelweave graft (Vascutek Terumo, Renfrewshire, Scotland) was then sewn to the artery in end-to-side fashion with a running continuous 6-0 polypropylene suture. The suture line was reinforced as necessary. The partially occluding crossclamp was then removed, the graft was de-aired, and satisfactory arterial flow was confirmed. The graft was then connected to the arterial return line. During the entire procedure, the near-infrared spectroscopy (NIRS) signal was carefully monitored, and the systemic blood pressure was maintained at 70 to $90 \mathrm{~mm} \mathrm{Hg}$. It is our practice to perform the anastomosis to the distal innominate artery close to the bifurcation. In cases in which the "take off" of the innominate artery is quite large and placement of a partial occlusion clamp is not considered safe, 2 atraumatic vascular clamps were placed with flow interruption of the innominate artery. Very rarely was complete interruption of flow necessary.

\section{Use of Innominate Artery Cannulation to Deliver ACP During Proximal Aortic Repair}

After the innominate artery was cannulated, a full dose of heparin was given $(3 \mathrm{mg} / \mathrm{kg}$ or $300 \mathrm{IU} / \mathrm{kg}$ ) with a target activated clotting time of 480 seconds or longer. Cardiopulmonary bypass was initiated, and the patient was cooled to $23^{\circ} \mathrm{C}$ to $24^{\circ} \mathrm{C}$. Ice was packed around the patient's head. Mannitol and hydrocortisone were also given to prevent cerebral edema, which can ensue during and after cooling. Once the target temperature was reached, the Rumel tourniquet around the proximal innominate artery was cinched down and systemic circulatory arrest initiated, during which time, selective ACP was delivered (target flow, $10-15 \mathrm{~mL} / \mathrm{kg} / \mathrm{min}$ ). Alternatively, an atraumatic vascular clamp was used to occlude the proximal innominate artery. After the transverse arch was visualized, a 9F Pruitt perfusion catheter (LeMaitre Vascular, Inc, Burlington, Mass), connected to the arterial line by a $\mathrm{Y}$-connector, was inserted into the left common carotid artery for left-sided cerebral perfusion. For most of the cases, the left subclavian artery was open to air. When the back flow from the left subclavian artery was profuse and interfered with visualization of the distal anastomosis, a balloon tip catheter was used to inhibit the forward flow. In no case was the left subclavian artery blocked as a specific response to changes in the NIRS. After the distal anastomosis was completed, the Rumel tourniquet or vascular clamp on the proximal innominate artery was removed, and the pump flow was slowly returned to its full level.

Once the Dacron graft had been de-aired and clamped, the proximal pathologic findings were assessed and addressed. We have previously described the use of the $Y$-graft to reconstruct the aortic arch. In the present series, however, we used innominate artery cannulation instead of right axillary cannulation. ${ }^{5}$ Cerebral perfusion pressure was monitored with NIRS, using the INVOS Cerebral/Somatic Oximeter (Somanetics, Troy, Mich) by way of a right radial arterial line, and was adjusted accordingly. Bifrontal electrophysiology monitoring as an index of gross cerebral perfusion was used occasionally, according to anesthesia preference. In all cases, we placed NIRS probes over the cranium to measure regional cerebral oxygen saturation. The pressure of antegrade cerebral perfusion was maintained at 50 to $70 \mathrm{~mm} \mathrm{Hg}$. Provided that we were already using bilateral ACP, a decrease in the NIRS regional cerebral oxygen saturation reading of more than $10 \%$ from the patient's baseline measurement prompted us to increase the flow (to $\geq 13-15 \mathrm{~mL} / \mathrm{kg} / \mathrm{min}$ ) and/or the arterial partial pressure of carbon dioxide. We use $\mathrm{pH}$ stat blood gas management, which tends to increase the arterial partial pressure of carbon dioxide and maintains cerebral vasodilation. We have not routinely used transcranial Doppler imaging and did not use it in the present study. Moreover, for assessing the global balance of cerebral oxygen supply and metabolic demand, jugular venous oximetry was used, in addition to NIRS, in a few patients in the present series who underwent total aortic arch replacement as a part of an ongoing neuromonitoring study.

\section{Statistical Analysis}

Statistical analysis was performed using SAS/STAT software, version 9.1.3, SAS System for Windows (SAS Institute Inc, Cary, NC). The first part was univariate analysis of the preoperative characteristics, operative variables, and postoperative outcomes. Data are reported as the median and interquartile range ( $25 \%$, quartile 1 , to $75 \%$, quartile 3 ) for continuous variables and as the number and percentage for categorical variables. In the second part of the analysis, we sought to determine which preoperative and operative characteristics might have increased the risk of postoperative stroke or temporary confusion. To extract as much information as possible from the limited data, we created a new dependent variable with a value of 0 for no postoperative neurologic event, 1 for temporary confusion, and 2 for stroke. We used a generalized linear model (GENMOD) in SAS and specified a multinomial distribution.

\section{RESULTS}

The results from the first part of the analysis are listed in Tables 1 through 4. The second part of the analysis and the results of the multinomial logistic model for neurologic events adjusted for preoperative and operative characteristics are listed in Table 5.

\section{Delivery of ACP}

Innominate artery cannulation using an 8-mm Dacron graft (Vascutek Terumo) was successful in all 68 patients. None of the patients had any significant change in their NIRS signal during cannulation, and no malperfusion issues were noted after the arterial line was connected to the side graft off the innominate artery. There was no need to change the cannulation site in any patient. Hypothermic circulatory 
TABLE 1. Indications for surgery $(n=68)$

\begin{tabular}{lc}
\hline \multicolumn{1}{c}{ Indication } & $\mathbf{n}(\%)$ \\
\hline Ascending aneurysm & $4(5.9)$ \\
Proximal arch aneurysm & $43(63.2)$ \\
With chronic dissection & 1 \\
Total arch aneurysm & $10(14.7)$ \\
$\quad$ With chronic dissection & 2 \\
Aortic dissection & $11(16.2)$ \\
Acute, subacute & 9 \\
Chronic & 2 \\
\hline
\end{tabular}

arrest with ACP was used in all cases that required open distal anastomosis $(\mathrm{n}=64$ patients; $94.1 \%)$. Of the 68 patients, $63(92.6 \%)$ received ACP to both cerebral hemispheres. The median antegrade perfusion time was 20 minutes (interquartile range, 15.0-33.0 minutes). Seven patients had periods of pure circulatory arrest without ACP for a median of 20 minutes (interquartile range, 6.0-33.0 minutes).

\section{Hospital Mortality}

One patient $(1.5 \%)$ died within 30 postoperative days. This patient was in New York Heart Association functional class III and had undergone a stage I elephant trunk procedure using the "island technique" and concomitant double coronary artery bypass, mitral valve repair, tricuspid valve repair, and cryomaze procedure. This patient developed profuse coagulopathy and septicemia and died on

TABLE 2. Preoperative characteristics $(n=68)$

\begin{tabular}{lc}
\hline \multicolumn{1}{c}{ Characteristic } & Value \\
\hline Age (y) & \\
$\quad$ Median & 61.5 \\
$\quad$ Range & $50.5-72.0$ \\
Male gender & $50(73.5)$ \\
Smoking & $59(86.8)$ \\
Hypertension & $59(86.8)$ \\
Cardiac disease unrelated to aorta & $32(47.1)$ \\
Bicuspid aortic valve & $16(23.5)$ \\
Acute, subacute aortic dissection & $9(13.2)$ \\
NYHA functional class & \\
$\quad$ I & $9(13.2)$ \\
II & $46(67.7)$ \\
III & $12(17.6)$ \\
IV & $1(1.5)$ \\
Genetically triggered thoracic aortic disease* & $6(8.8)$ \\
Pulmonary disease & $19(27.9)$ \\
Renal dysfunction & $9(13.2)$ \\
Previous CBV disease & $8(11.8)$ \\
Diabetes mellitus & $5(7.4)$ \\
Alcohol/substance abuse & $9(13.2)$ \\
\hline Data presented as median and interquartile range (25\%, quartile 1 to $75 \%$, quartile 3$)$ \\
for continuous variables and n (\%) for categorical variables. NYHA, New York Heart \\
Association; $C B V$, cerebrovascular. *Marfan disease (n = 4), family history $(\mathrm{n}=1)$, \\
and genetic mutation (n = 1).
\end{tabular}

TABLE 3. Operative variables

\begin{tabular}{lc}
\multicolumn{1}{c}{ Variable } & Value \\
\hline Repeat sternotomy & $6(8.8)$ \\
Urgency of operation & \\
Elective & $59(86.7)$ \\
Emergency & $8(11.8)$ \\
Urgent & $1(1.5)$ \\
Index procedure & \\
Proximal arch or hemiarch repair & $52(76.5)$ \\
Total arch repair & $12(17.6)$ \\
Ascending aortic repair & $4(5.9)$ \\
Concomitant procedures & \\
Aortic root replacement & $22(32.4)$ \\
Aortic valve repair & $20(29.4)$ \\
Aortic valve replacement & $16(23.5)$ \\
Endovascular repair of DTA (TEVAR) & $12(17.6)$ \\
Coronary artery bypass & $11(16.2)$ \\
Aortic valve sparing root replacement & $3(4.4)$ \\
Tricuspid, mitral repair & $1(1.5)$ \\
Other & $6(8.8)$ \\
Operative time (min) & \\
CPB & $120.5(84.5-145.5)$ \\
Myocardial ischemia time & $84.0(55.0-103.0)$ \\
ACP time (n = 64) & $20.0(15.0-33.0)$ \\
Cerebral circulatory arrest time (n $=7)$ & $20.0(6.0-33.0)$ \\
Duration of surgery & $351.0(295.5-414.5)$ \\
\hline Dat & \\
\end{tabular}

Data are presented as median (interquartile range; $25 \%$, quartile 1 to $75 \%$, quartile 3 ) for continuous variables and $\mathrm{n}(\%)$ for categorical variables. DTA, Descending thoracic aorta; TEVAR, thoracic endovascular aortic repair; $C P B$, cardiopulmonary bypass; $A C P$, antegrade cerebral perfusion.

postoperative day 7 . Two patients died after 30 days but while still in the hospital. The first patient was in New York Heart Association class III and had undergone total arch replacement with a stented elephant trunk and triple coronary artery bypass. This patient experienced a stroke and developed multiorgan failure, and the family gave permission for life support to be withdrawn on postoperative day 36. The other nonsurvivor was in New York Heart Association class II and had undergone aortic valve

TABLE 4. Early outcomes other than neurologic events $(n=68)$

\begin{tabular}{lc}
\hline \multicolumn{1}{c}{ Outcome } & Patients (n) \\
\hline 30-Day mortality & $1(1.5)$ \\
In-hospital mortality & $3(4.4)$ \\
Tracheostomy & $8(11.8)$ \\
Atrial fibrillation & $29(42.7)$ \\
Acute renal insufficiency* & $5(7.4)$ \\
Reoperation for bleeding & $5(7.4)$ \\
Poor wound healing/dehiscence & $3(4.4)$ \\
Pericardial window & $2(2.9)$ \\
Deep venous thrombosis & $2(2.9)$ \\
Heparin-induced thrombocytopenia & $2(2.9)$ \\
Intensive care unit stay (d) & $3(2.0-8.5)$ \\
\hline Dan par
\end{tabular}

Data in parentheses are percentages. *Two of these patients $(2.9 \%)$ required temporary hemodialysis; none required permanent hemodialysis. 
TABLE 5. Multinomial model for predicting neurologic events

\begin{tabular}{lcc}
\hline \multicolumn{1}{c}{ Variable } & Estimated OR $(\mathbf{9 5} \%$ CI $)$ & $\boldsymbol{P}$ value \\
\hline Previous CBV disease & $2.45(0.13-45.17)$ & .52 \\
Repeat sternotomy & $2.26(0.15-35.01)$ & .55 \\
Surgery type* & $0.11(0.004-3.00)$ & .15 \\
Age & $1.08(1.01-1.17)$ & .02 \\
Myocardial ischemia time & $0.99(0.96-1.03)$ & .70 \\
ACP time & $1.06(1.01-1.11)$ & .01 \\
CPB time & $1.00(0.99-1.02)$ & .79 \\
Gender & $1.41(0.25-7.87)$ & .70 \\
\hline
\end{tabular}

$O R$, Odds ratio; $C I$, confidence interval; $C B V$, cerebrovascular; $A C P$, antegrade cerebral perfusion; $C P B$, cardiopulmonary bypass. *Proximal aneurysm repair vs total arch aneurysm repair.

replacement with a tissue valve and ascending and proximal arch repair. This patient had a prolonged hospital course complicated by multiorgan failure. The family approved the discontinuation of life support, and the patient died on postoperative day 120 .

\section{Neurologic Events}

Three patients $(4.4 \%)$ had strokes, including the previously mentioned patient who died on postoperative day 36. During surgery, he had a prolonged ACP time (123 minutes) with no cerebral circulatory arrest time. The other 2 patients had minor strokes while still in the hospital and underwent significant, although partial, recovery. One of these strokes occurred in a patient with an ascending aortic aneurysm who presented with a proximal type I endoleak and localized retrograde dissection secondary to thoracic endovascular stent placement for a descending thoracic aneurysm. The patient underwent repair of the ascending aorta and hybrid repair of the total aortic arch, and the antegrade cerebral perfusion time by bilateral delivery of ACP was 86 minutes. Postoperatively, this patient developed bilateral cerebellar infarcts; however, the neurologic status improved significantly before hospital discharge. The second patient was an 86-year-old woman who had undergone proximal arch repair and single coronary artery bypass. Her ACP time was 20 minutes with no period of cerebral circulatory arrest. Her neurologic status had significantly improved before hospital discharge.

Seven patients $(10.3 \%)$ had postoperative confusion (transient disturbances of consciousness or altered cognition). The confusion resolved within 3 days in 4 patients and after 1 week in the other 3 patients. The neurologic condition of the patients was evaluated daily by the operative team, including a critical care specialist dedicated to the team. Only patients with strokes were evaluated by a neurologist. No cases of paraplegia developed. One patient $(1.5 \%)$ who had undergone stage I stented elephant trunk developed transient paraparesis within the first 24 hours, but it resolved immediately after cerebrospinal fluid drainage.

\section{Early Outcomes Other Than Neurologic Conditions}

The early outcomes, other than neurologic conditions, are listed in Table 4 . None of the patients had any vascular injuries or infection at the innominate artery cannulation site.

\section{DISCUSSION}

In aortic surgery, the most widely used cannulation strategies are direct ascending aortic and femoral artery cannulation and direct axillary artery cannulation or indirect cannulation using a side graft; cannulation of the innominate artery is a less common approach. ${ }^{1,4-10}$ The optimal cannulation site is a subject of continuous debate among experienced aortic surgical groups. ${ }^{6,11,12}$ In patients with severe atherosclerotic disease, direct aortic cannulation can cause serious embolization. Moreover, in acute type 1 aortic dissection, direct aortic cannulation can cause malperfusion or extension of the dissection. Despite these risks, the safety of direct aortic cannulation has been confirmed by several groups. ${ }^{13,14}$

When cannulation of the ascending aorta poses a prohibitive risk, femoral artery cannulation is often used. Experienced surgeons routinely use femoral artery cannulation for thoracic aortic surgery with excellent results. ${ }^{6}$ Nevertheless, in cases of acute type 1 aortic dissection, femoral artery cannulation can result in malperfusion. ${ }^{7,11}$ Also, in patients with descending and extensive thoracoabdominal aneurysms, femoral cannulation can increase the risk of aortic dissection and retrograde embolization secondary to dislodged atheromas. ${ }^{7,8,11}$ These complications, along with the inability to deliver ACP, have led to the widespread use of axillary artery cannulation as an alternative approach in patients with ascending aortic and arch pathologic findings. ${ }^{1,7,8,11,15}$ A key advantage of axillary artery cannulation is the ability to deliver ACP, which allows complex aortic repair. ${ }^{5,11}$

At our institution, the cannulation strategies for proximal aortic surgery have evolved toward the routine use of axillary artery cannulation in elective and emergent cases with good outcomes. ${ }^{1,5}$ Although axillary artery cannulation is usually well tolerated, various complications have been reported, including brachial plexus injury, localized dissection, arm ischemia, inadequate cardiopulmonary flow, and malperfusion. ${ }^{16-18}$ Sabik and associates ${ }^{19}$ suggested that use of a side graft for axillary artery cannulation might reduce morbidity. Another alternative cannulation site is the innominate artery; however, the published data contain fewer reports about this approach than about axillary or femoral cannula-

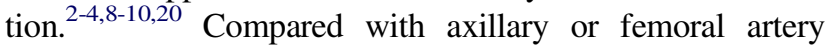
cannulation, innominate artery cannulation has several advantages. First, there is no need for an additional incision; thus, the operative time is potentially shorter. Second, because the cannulation site remains under the surgeon's 
direct vision at all times, additional blood loss and possible kinking of the cannula can be avoided. Third, cannulation is technically easier to perform in obese patients. Fourth, the risk of brachial plexus injury, arm claudication, or ischemia (associated with axillary cannulation) is eliminated. Fifth, unlike femoral cannulation, innominate artery cannulation prevents malperfusion and retrograde cerebral atheroembolism and provides delivery of ACP at all times. Finally, postoperatively, patients can undergo early ambulation and physical therapy (which might not be possible with femoral cannulation).

Since June 2011, we have used innominate artery cannulation in proximal aortic repairs when we thought this approach would be safe and appropriate. Manipulations around the innominate artery are not without risk, because the phrenic nerve and sympathetic chain might be close, depending on the anatomy. We did not encounter any injury or bleeding in any of our patients with regard to the cannulation site. Knowledge of the anatomic structures is imperative. We have shown that innominate artery cannulation is safe in a variety of proximal aortic procedures and does not compromise the ability to provide ACP.

In cases of redo sternotomy, a preoperative computed tomography scan of the chest was necessary to evaluate the proximity of the heart and aorta to the sternum, ensuring safe performance of a median sternotomy. If the aorta was adherent to the posterior sternum or was in close proximity to it, an alternative to innominate cannulation for arterial inflow was chosen before performing the median sternotomy. Our second choice was to cannulate the right axillary artery using a side graft, leaving femoral artery cannulation as the last choice. In hemodynamically unstable patients in whom cardiopulmonary bypass needs to be initiated rapidly, femoral cannulation is an appealing option, depending on the patient's body habitus.

Other surgical groups have also obtained excellent results with innominate artery cannulation. Huang and colleagues ${ }^{2}$ used a side graft technique to cannulate the innominate artery in 46 patients. Postoperatively, 5 of the patients $(10.9 \%)$ had temporary neurologic dysfunction, just as in our series. No stroke was reported, and the 30-day mortality rate was $6.5 \%$. In 55 patients, Di Eusanio and colleagues ${ }^{4}$ used a similar side graft technique to cannulate the innominate artery and used ACP during circulatory arrest. One patient $(1.8 \%)$ had transient neurologic dysfunction, and the overall hospital mortality rate was $3.6 \%$. In contrast, $\mathrm{Ji}$ and colleagues ${ }^{9}$ cannulated the innominate artery directly in 68 patients. The cannula tip was oriented toward the aortic arch during the initial period of cooling and final rewarming, and it was turned gently toward the patient's head during systemic circulatory arrest. No neurologic event was reported, and the 30-day mortality was $2.9 \%$.

To eliminate the sandblasting effect of turbulent flow from a catheter tip cannula close to an atherosclerotic or a dissected arch and to prevent dissection at the cannulation site, all the patients in our series had a side graft attached to the innominate artery. It is our distinct clinical impression that innominate artery cannulation contributed to the neurologic events in 2 of our patients with arch replacement. We believe their prolonged antegrade cerebral perfusion time (123 and 86 minutes, respectively), required by the extent of their disease and the anatomic complexity of the reconstruction, led to the neurologic injury $(P=.01$; Table 5). In the case of the third patient, who experienced a smaller stroke with partial recovery, we believe that her advanced age contributed to the neurologic event $(P=.02$; Table 5). In analyzing 1336 operations, including those performed with femoral artery cannulation, Svensson and colleagues ${ }^{8}$ encountered a $4.2 \%$ stroke rate with direct innominate cannulation without a side graft ( 24 patients) compared with a $4 \%$ stroke rate with axillary cannulation using a side graft (299 patients) and a 7.8\% stroke rate with direct cannulation (167 patients). ${ }^{8}$ Retrograde brain perfusion was used in 933 patients $(69 \%)$. We have not used retrograde cerebral perfusion in any of our patients.

Etz and associates, ${ }^{11}$ at Mount Sinai Hospital, reported their 10-year experience with 869 patients who underwent ascending aorta or root repairs for atheromatous and degenerative aneurysms and type A dissections. These surgeons compared aortic cannulation $(\mathrm{n}=157)$ with femoral $(\mathrm{n}=261)$ and direct right axillary $(\mathrm{n}=451)$ cannulation. The stroke and mortality rates were superior with right axillary cannulation. Our group previously reported the results of right axillary cannulation in 83 patients with acute or subacute ascending aortic dissection; the stroke rate was $11 \%$, and the 30-day (hospital) mortality was $17 \% .{ }^{1}$ In 86 patients with acute type A dissection, Fusco and associates ${ }^{21}$ encountered the same mortality rate $(17 \%)$ and an $8 \%$ perioperative stroke rate with femoral cannulation. Eleven of our patients had ascending aortic dissections, including nine acute dissections, and underwent innominate artery cannulation. No 30-day mortality occurred in this subgroup, and 1 patient experienced stroke with partial recovery. In none of our patients with aortic dissection was the innominate artery dissected before cannulation.

With regard to the study limitations, our study was observational and performed exclusively on patients in whom innominate artery cannulation was used. We had no intent to compare this site with other cannulation sites.

\section{CONCLUSIONS}

Innominate artery cannulation is a safe, effective alternative to femoral or axillary cannulation for arterial inflow in proximal aortic surgery. An individualized approach is critical to ensure patient safety.

The authors thank Katherine H. Simpson for help with the statistical analyses, and Virginia C. Fairchild, Department of 
Scientific Publications, Texas Heart Institute, for editorial assistance in preparing our report.

\section{References}

1. Wong DR, Coselli JS, Palmero L, Bozinovski J, Carter SA, Murariu D, et al. Axillary artery cannulation in surgery for acute or subacute ascending aortic dissections. Ann Thorac Surg. 2010;90:731-7.

2. Huang FJ, Wu Q, Ren CW, Lai YQ, Yang S, Rui QJ, et al. Cannulation of the innominate artery with a side graft in arch surgery. Ann Thorac Surg. 2010;89: 800-3.

3. Banbury MK, Cosgrove DM III. Arterial cannulation of the innominate artery. Ann Thorac Surg. 2000;69:957.

4. Di Eusanio M, Ciano M, Labriola G, Lionetti G, Di Eusanio G. Cannulation of the innominate artery during surgery of the thoracic aorta: our experience in 55 patients. Eur J Cardiothorac Surg. 2007;32:270-3.

5. LeMaire SA, Price MD, Parenti JL, Johnson ML, Lay AD, Preventza O, et al. Early outcomes after aortic arch replacement by using the Y-graft technique. Ann Thorac Surg. 2011;91:700-7; discussion 707-8.

6. Ayyash B, Tranquilli M, Elefteriades JA. Femoral artery cannulation for thoracic aortic surgery: safe under transesophageal echocardiographic control. $J$ Thorac Cardiovasc Surg. 2011;142:1478-81.

7. Strauch JT, Spielvogel D, Lauten A, Lansman SL, McMurtry K, Bodian CA, et al. Axillary artery cannulation: routine use in ascending aorta and aortic arch replacement. Ann Thorac Surg. 2004;78:103-8; discussion 103-8.

8. Svensson LG, Blackstone EH, Rajeswaran J, Sabik JF III, Lytle BW, GonzalezStawinski G, et al. Does the arterial cannulation site for circulatory arrest influence stroke risk? Ann Thorac Surg. 2004;78:1274-84; discussion 1274-84.

9. Ji S, Yang J, Ye X, Wang X. Brain protection by using innominate artery cannulation during aortic arch surgery. Ann Thorac Surg. 2008;86:1030-2.

10. Chiu KM, Li SJ, Lin TY, Chan CY, Chu SH. Innominate artery cannulation for aortic surgery. Asian Cardiovasc Thorac Ann. 2007;15:348-50.
11. Etz CD, Plestis KA, Kari FA, Silovitz D, Bodian CA, Spielvogel D, et al. Axillary cannulation significantly improves survival and neurologic outcome after atherosclerotic aneurysm repair of the aortic root and ascending aorta. Ann Thorac Surg. 2008;86:441-6; discussion 446-7.

12. Tiwari KK, Murzi M, Bevilacqua S, Glauber M. Which cannulation (ascending aortic cannulation or peripheral arterial cannulation) is better for acute type A aortic dissection surgery? Interact Cardiovasc Thorac Surg. 2010;10:797-802.

13. Minatoya K, Karck M, Szpakowski E, Harringer W, Haverich A. Ascending aortic cannulation for Stanford type A acute aortic dissection: another option. J Thorac Cardiovasc Surg. 2003;125:952-3.

14. Reece TB, Tribble CG, Smith RL, Singh RR, Stiles BM, Peeler BB, et al. Central cannulation is safe in acute aortic dissection repair. J Thorac Cardiovasc Surg. 2007;133:428-34.

15. Sabik JF, Lytle BW, McCarthy PM, Cosgrove DM. Axillary artery: an alternative site of arterial cannulation for patients with extensive aortic and peripheral vascular disease. J Thorac Cardiovasc Surg. 1995;109:885-90; discussion 890-1.

16. Orihashi K, Sueda T, Okada K, Takahashi S. Compressed true lumen in the innominate artery: a pitfall of right axillary arterial perfusion in acute aortic dissection. J Thorac Cardiovasc Surg. 2009;137:242-3.

17. Rescigno G, Aratari C, Matteucci ML. Axillary artery cannulation pitfalls. J Thorac Cardiovasc Surg. 2009;138:251-2.

18. Schachner T, Nagiller J, Zimmer A, Laufer G, Bonatti J. Technical problems and complications of axillary artery cannulation. Eur J Cardiothorac Surg. 2005;27: 634-7.

19. Sabik JF, Nemeh H, Lytle BW, Blackstone EH, Gillinov AM, Rajeswaran J, et al. Cannulation of the axillary artery with a side graft reduces morbidity. Ann Thorac Surg. 2004;77:1315-20.

20. Augoustides JGT, Harris H, Pochettino A. Direct innominate artery cannulation in acute type A dissection and severe thoracic aortic atheroma. J Cardiothorac Vasc Anesth. 2006;21:727-9.

21. Fusco DS, Shaw RK, Tranquilli M, Kopf GS, Elefteriades JA. Femoral cannulation is safe for type A dissection repair. Ann Thorac Surg. 2004;78:1285-9; discussion 1285-9. 